\title{
Development of an atmospheric climate model with self-adapting grid and physics
}

\author{
Joyce E. Penner ${ }^{1}$, Michael Herzog ${ }^{2}$, Christiane Jablonowski ${ }^{3}$, Bram van Leer ${ }^{1}$, \\ Robert C. Oehmke ${ }^{1}$, Quentin F. Stout ${ }^{1}$, and Kenneth G. Powell ${ }^{1}$ \\ ${ }^{1}$ University of Michigan \\ ${ }^{2}$ Geophysical Fluid Dynamics Laboratory \\ ${ }^{3}$ National Center for Atmospheric Research \\ penner@umich.edu
}

\begin{abstract}
An adaptive grid dynamical core for a global atmospheric climate model has been developed. Adaptations allow a smooth transition from hydrostatic to non-hydrostatic physics at small resolution. The adaptations use a parallel program library for block-wise adaptive grids on the sphere. This library also supports the use of a reduced grid with coarser resolution in the longitudinal direction as the poles are approached. This permits the use of a longer time step since the CFL number restriction $(\mathrm{CFL}<1)$ in a regular longitude-latitude grid is most severe in the zonal direction at high latitudes. Several tests show that our modelling procedures are stable and accurate.
\end{abstract}

\section{Introduction}

The goal of this research project is to develop adaptive grid techniques for future climate model and weather predictions. This approach will lead to new insights into small-scale and large-scale flow interactions that are unresolved by current uniform-grid simulations. Adaptive mesh refinement (AMR) techniques provide an attractive framework for atmospheric motions since they allow improved horizontal resolution in a limited region without requiring a fine grid resolution throughout the entire model domain. Therefore, the model domain to be resolved with higher resolution is kept at a minimum, greatly reducing computer memory and speed requirements.

Adaptive grid techniques have been developed for a parallel version of the NASA / NCAR FiniteVolume Community Climate Model. This global hydrostatic model is based on NCAR physics and the so-called Lin-Rood finite-volume dynamical core ${ }^{1}$ that provides highly efficient algorithms for high performance computing.

This research project is characterized by an interdisciplinary approach involving atmospheric science, computer science and mathematical/numerical aspects. The work is done in close collaboration between the Atmospheric Science, Computer Science and Aerospace Engineering Departments at the University of Michigan, NCAR, and NASA.

\section{The Adaptive Grid Library}

The newly developed version of the NASA finite-volume dynamical core with self-adaptive grid uses a general purpose library of modules to implement the block-wise adaptive grids on the sphere. As 
indicated in Figure 1, the grid is subdivided horizontally into self-similar blocks that contain an identical number of grid points per block. In the event of a refinement a block is split into four new blocks, thereby doubling the spatial resolution; coarsening reverses this process. Here the spatial resolution of adjacent blocks is only allowed to differ by a factor of two. On the sphere, a regular longitude-latitude grid has been adopted. In addition, an initial reduced grid setup can be selected (Figure 2). In case the reduced grid is selected, the longitudinal resolution in polar regions is coarsened which alleviates the convergence of the meridians at the poles.

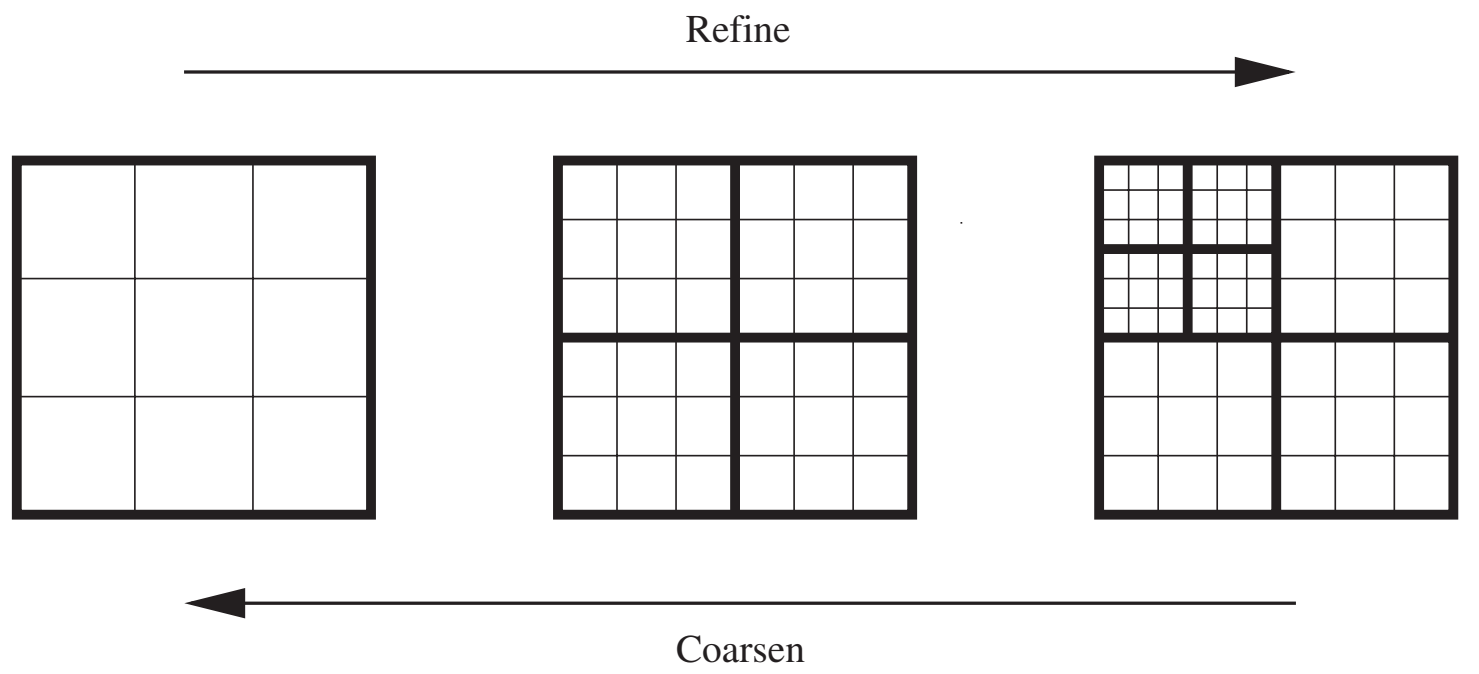

Figure 1: Schematic view of the refinement and coarsening principles with 2 refinement levels and 3 $\times 3$ grid cells per block.
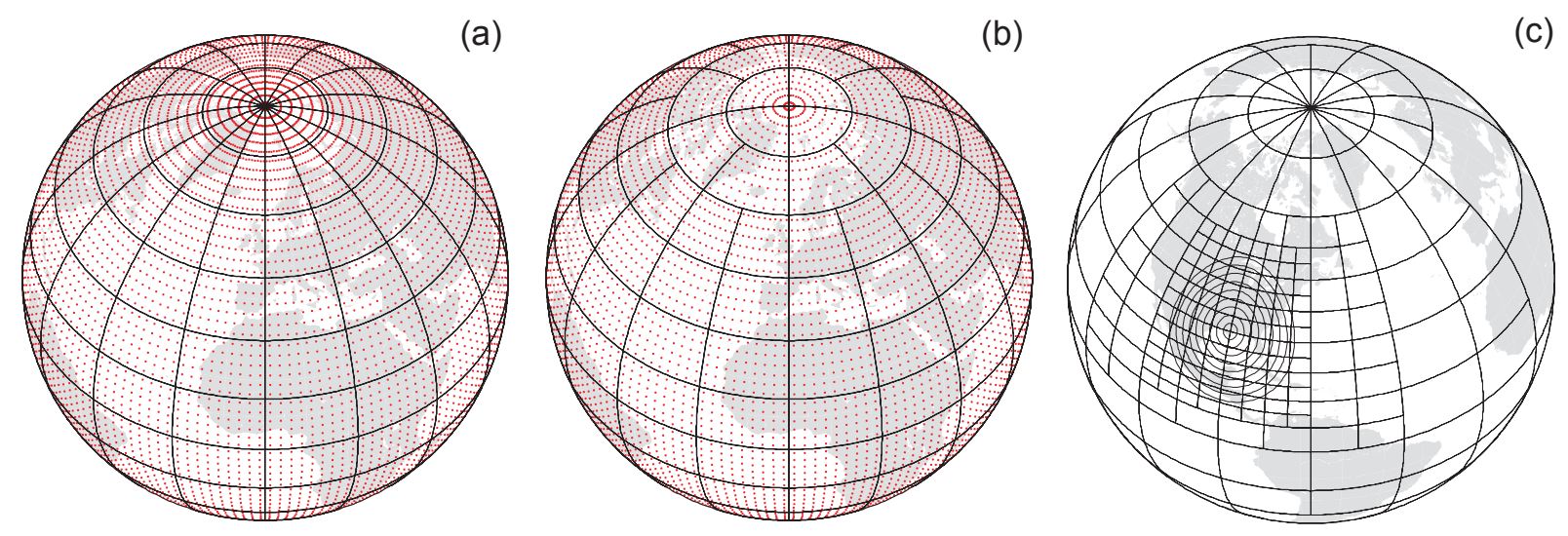

Figure 2: Distribution of grid points and blocks over the sphere in an orthographic projection centered at $\left(45^{\circ} \mathrm{N}, 0^{\circ}\right)$. The resolution is $2.5^{\circ} \times 2.5^{\circ}$ (a) non-adapted case, (b) reduced grid case, (c) reduced grid showing adaptations.

The parallel adaptive grid library provides the functionality necessary to implement the grid data structures by providing the following services: 1) the creation of the initial spherical grid including setting up neighbor connections, and distributing blocks across processors, 2) the update of ghost cells transparent to processor location, 3) the adaptation of blocks including providing communication for user-data update, maintaining neighbor connections and redistributing blocks. The library was designed for flexibility and efficiency, allowing the user to arrange their data however they wish. User-defined subroutines need to be provided that specify the algorithms for split and join, and ghost 
cell operations. These routines include the interpolation and averaging procedures for the initialization of new blocks and the data exchange algorithms for neighboring blocks at both identical and varying resolutions.

\section{The Hydrostatic Dynamical Core}

Statically and dynamically adaptive grids have been successfully implemented and tested in 2D shallow water simulations and 3D hydrostatic dynamical core runs on the sphere. Figure 3 shows an example of a 2D shallow water simulation at model day 10. The depicted geopotential height field is characterized by a lee-side wave that is induced by an idealized mountain. Here a combination of statically and dynamically refined blocks is presented. The dynamic adaptations track the evolution of the wave by a gradient-based adaptation criterion. Other adaptation criteria that are, for example, based on vorticity have also been successfully applied.

Tests with the adaptive 3D hydrostatic dynamical core suggest that adaptations are a viable option for future modeling studies. 3D idealized experiments with locally refined resolutions along storm tracks have been performed. The results show that the developing storm systems are predicted accurately without the need for a fine resolution in the entire model domain.

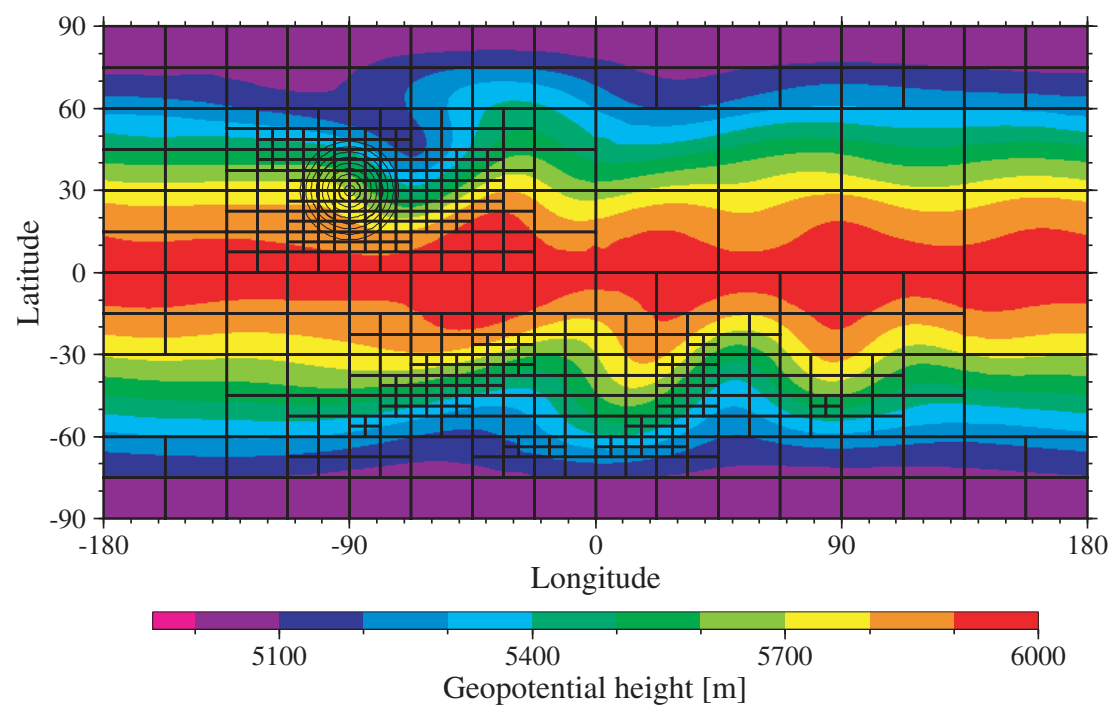

Figure 3: Geopotential height field at day 10.

\section{The Non-Hydrostatic Dynamical Core}

One of the most important advances needed in global climate models is the development of models that can reliably treat convection. At the present time, convection is a sub-grid process that must be parameterized.

The explicit treatment of convection requires spatial resolutions at which the hydrostatic assumption is no longer valid. Therefore we have developed a non-hydrostatic code which smoothly replaces the hydrostatic treatment when required. In the non-hydrostatic formulation, a mass-based Lagrangian vertical coordinate replaces the pressure-based Lagrangian vertical coordinate of the hydrostatic code. In this system, the mass continuity equation is:

$$
\left(\frac{\partial \pi}{\partial t}\right)_{s}+\nabla_{s}(\vec{v} \pi)+\frac{\partial(\dot{s} \pi)}{\partial s}=0
$$


where $\dot{s}=\frac{d s}{d t}$ is the vertical velocity and $\pi=-\rho g \frac{\partial z}{\partial s}$ is the vertically integrated mass per unit area. Our Lagrangian vertical coordinate is defined by $\dot{s}=0$, so that the last term in (1) is zero.

Prognostic equations for the density anomaly and the vertical velocity anomaly are solved. Thus, the anomaly (from the hydrostatic equilibrium solution) of the density, $\rho^{\prime}$, and the anomaly of the vertical velocity, $w^{\prime}$, are predicted from:

$$
\begin{gathered}
\left(\frac{\partial \rho^{\prime}}{\partial t}\right)_{s}+\nabla_{s}\left(\rho^{\prime} \vec{v}\right)=2 \frac{\tilde{\rho} \rho^{\prime}}{\pi}\left(g \frac{\partial \tilde{w}}{\partial s}-\nabla_{s} \tilde{\Phi} \frac{\partial \vec{v}}{\partial s}\right)+\frac{\tilde{\rho}^{2}}{\pi}\left(g \frac{\partial w^{\prime}}{\partial s}-\left(\nabla_{s} \Phi^{\prime}\right) \frac{\partial \vec{v}}{\partial s}\right) \\
\left(\frac{\partial}{\partial t}\left(\pi w^{\prime}\right)\right)_{s}+\nabla_{s}\left(\pi w^{\prime} \vec{v}\right)-g \frac{\partial P^{\prime}}{\partial s}=0
\end{gathered}
$$

where $\tilde{\rho}, \tilde{w}$, and $\tilde{\Phi}$ are the hydrostatic solutions for the density, vertical velocity and geopotential, and $\vec{v}$ is the horizontal wind vector. The full density $\rho=\tilde{\rho}+\rho^{\prime}$ is used to predict the full geopotential, $\Phi$, from which the non-hydrostatic geopotential anomaly is derived, and the hydrostatic vertical velocity $\tilde{w}$ is derived from the temporal evolution of $\tilde{\Phi} . P^{\prime}$ is calculated from the hydrostatic and non-hydrostatic densities, $\rho$ and $\rho^{\prime}$.

\subsection{Upper boundary conditions}

Along the characteristic $d\left(P^{\prime}-\rho c_{s} w\right)$ equals zero. Here, $P^{\prime}$ is the non-hydrostatic pressure anomaly, $\rho$ the density, $c_{s}$ the speed of sound and $\mathrm{w}$ the vertical velocity. Assuming equilibrium and constant density, the above characteristic reduces in one dimension to a condition for the vertical gradient:

$$
\frac{\partial w}{\partial z}=\frac{1}{\rho c_{s}} \frac{\partial P^{\prime}}{\partial z}
$$

The use of Equ. (4) for the upper boundary condition accounts for the coupling between the anomalies for pressure and vertical velocity at the model top. However, the atmosphere is not in equilibrium and the vertical density gradient is far from being zero. Starting from an initial nonhydrostatic density (or pressure) perturbation, we simulated the hydrostatic adjustment process applying equation (4) at the model top. The model failed to reach hydrostatic equilibrium, and strong reflections occurred at the model top. The model was numerically unstable.

To take into account the transitional character of the atmosphere, we formulated an upper boundary condition based on time derivatives:

$$
\frac{\partial w}{\partial t}=\frac{1}{\rho c_{s}} \frac{\partial P^{\prime}}{\partial t}
$$

With this condition the model is much better behaved. Since equation (5) assumes constant density and doesn't do a full backward integration along the characteristic, small reflections of sound waves still occur at the model top (see Figure 4a).

To ensure that hydrostatic equilibrium is always reached, we added a damping term for $P^{\prime}$ in equation (5):

$$
\frac{\partial w}{\partial t}=\frac{1}{\rho c_{s}}\left(\frac{\partial P^{\prime}}{\partial t}\right)-\frac{P^{\prime}}{\tau}
$$


where $\tau$ is a time constant on the order of several minutes. This procedure has greatly reduced reflections at the model top, but has not entirely eliminated them (Figure $4 \mathrm{~b}$ ). Defining the vertical velocity at the cell interfaces in the vertical direction, rather than at the cell center, facilitates the implementation of a semi-implicit time-stepping algorithm, which remains stable at longer time steps and damps the fast-traveling sound waves (Figure 4c).

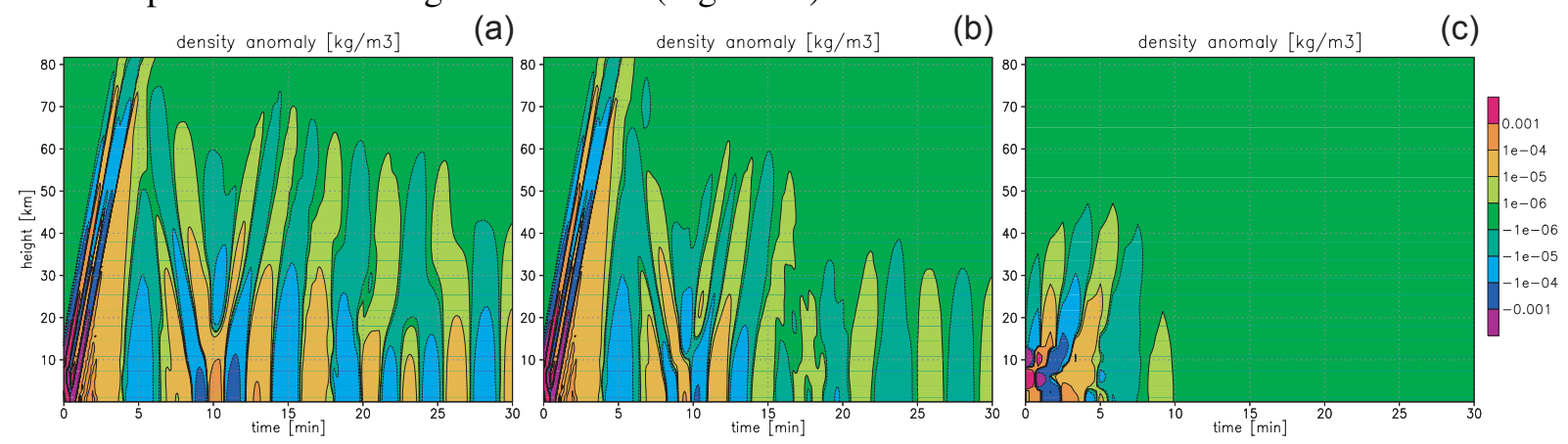

Figure 4: Propagation of a density anomaly in the non-hydrostatic code. The anomaly is initiated near $5 \mathrm{~km}$ and propagates vertically where it is reflected at the upper boundary. (a) shows an explicit time step procedure with no damping. (b) shows the explicit model with damping added. (c) shows an implicit version with no damping.

\section{Conclusions}

This project is aimed at developing a climate model that self-adjusts the grid resolution and the complexity of the physics model to the actual atmospheric flow conditions. To accomplish this, we have implemented a fully 3-dimensional non-hydrostatic model within a hydrostatic code while using a block-structured grid that allows for the implementation of smaller grid resolution within both the hydrostatic and non-hydrostatic portions of the grid. Several tests of the model show that it is stable and can smoothly transition from hydrostatic to non-hydrostatic flows.

One of the major building blocks of this project is a parallel adaptive grid library which is currently under development at the University of Michigan under the direction of co-I Professor Stout. This MPI-based communication library manages the block-structured data layout, handles the ghost cell updates among neighboring blocks and splits a block as refinements occur. The current functionality provides routines for a reduced grid design. In a reduced grid, the resolution is coarsened in the longitudinal direction as the pole is approached. This often allows the use of a longer time step since the CFL number restriction $(\mathrm{CFL}<1)$ in a regular longitude-latitude grid is most severe in the zonal direction at high latitudes. We have recently submitted a paper describing the use of this library in the hydrostatic portion of our code for advection. ${ }^{2}$

\section{References}

[1] Lin, S.-J. and R. B. Rood, 1996: Multidimensional flux-form semi-Lagrangian transport scheme, Mon. Wea. Rev., 124, 2046-2070.

[2] Jablonowski, C., M. Herzog, J.E. Penner, R.C. Oehmke, Q.F. Stout, B. van Leer, and K.G. Powell, 2005: Block-Structured Adaptive Grids on the Sphere: Advection Experiments, Mon. Wea. Rev., submitted. 